\title{
Impact of content characteristics and emotion on behavioral engagement in social media: literature review and research agenda
}

\author{
Melanie Schreiner ${ }^{1}$ (D) Thomas Fischer $^{1} \cdot$ Rene Riedl $^{1,2}$
}

Published online: 10 May 2019

(c) The Author(s) 2019

\begin{abstract}
We present a review of $\mathrm{N}=45$ studies, which deals with the effect of characteristics of social media content (e.g., topic or length) on behavioral engagement. In addition, we reviewed the possibility of a mediating effect of emotional responses in this context (e.g., arousing content has been shown to increase engagement behavior). We find a diverse body of research, particularly for the varying content characteristics that affect engagement, yet without any conclusive results. We therefore also highlight potential confounding effects causing such diverging results for the relationship between content characteristics and content engagement. We find no study that evaluates the mediating effect of emotional responses in the content-engagement relationship and therefore call for further investigations. In addition, future research should apply an extended communication model adapted for the social media context to guarantee rigorous research.
\end{abstract}

Keywords Affective content · Content engagement · Content marketing · Emotional effect $\cdot$ Engagement behavior $\cdot$ Social media communication

\section{Introduction}

Being present on social media platforms such as Facebook, Twitter, or Instagram has become important for companies for a variety of reasons, be it to promote their brand, their products, or for general publicity [2]. It is estimated that by 2019 , the content marketing industry will have a volume of about $\$ 300$ billion

Electronic supplementary material The online version of this article (https://doi.org/10.1007/s 1066 0-019-09353-8) contains supplementary material, which is available to authorized users.

Melanie Schreiner

melanie.schreiner@fh-steyr.at

1 University of Applied Sciences Upper Austria, Steyr, Austria

2 Johannes Kepler University Linz, Linz, Austria 
and thus will have more than doubled its volume within only 5 years (2014: about $\$ 145$ billion) [63]. User interaction with this content (i.e., content engagement) is a key indicator of its popularity and is used to assess the success of social media activities (e.g., [8]). Moreover, content engagement is a precondition for the positive impact of social media content on company success (e.g., "likes" of a company's Facebook post may have a positive effect on sales performance [21], or branding [22]). Hence, we initially want to summarize research on content characteristics by answering the following question:

RQ 1. Which content characteristics have been shown to be positively related to engagement?

In addition to the direct link between content characteristics and engagement, it has also been shown that social media activities can elicit emotional responses. For example, based on emotion self-reports, Lin and Utz [34] evaluated the positive (i.e., happiness) and negative (i.e., envy) emotional response when browsing through Facebook messages. Their results show that a close relationship to the sender of the post evokes more happiness. Further, using a range of neurophysiological measures (i.e., skin conductance, pupil dilation, blood volume pulse, respiratory activity, electromyogram, electroencephalogram) Mauri et al. [37] compared the experience of viewing one's own Facebook page with relaxing or stressful activities (i.e., viewing a slide show of panoramas or mathematical tasks). They found clear neurophysiological evidence for a flow state elicited by social media interaction, which is significantly different from relaxation or stress and characterized by high arousal and high valence (i.e., a very positive and exciting experience).

This study also highlights the conceptualization of the relationship between content (input) and engagement (behavior) as we want to apply it in this review, which is in accordance with the stimulus-organism-response ( $-\mathrm{O}-\mathrm{R}$ ) paradigm [62]. Based on this conceptualization, we are interested in the general effect of content as an input on engagement as an output, mediated by individual affective processes (organism), which are represented by emotional responses. This leads us to the second research question:

RQ 2. Which effects do emotions have in the context of content characteristics and content engagement?

In line with traditional procedures for the analysis of mediator effects (e.g., [5]), we further break down this research question into three additional sub-questions; that is: RQ 2a on the content/emotion relationship, RQ $2 \mathrm{~b}$ on the emotion/engagement relationship, and we further include RQ $2 \mathrm{c}$ to report on studies which have already investigated a potential mediating effect:

RQ 2a. Which effects do content characteristics have on emotions?

RQ 2b. Which effects do emotions have on content engagement?

RQ 2c. Has there been research into the mediating effect of emotions between content characteristics and content engagement? 
The resulting research model, which will guide our literature review, is summarized in Fig. 1. Based on the involved relationships, the remainder of this article is structured as follows: In the next section, we discuss our understanding of content engagement, content characteristics, and emotions, and present working definitions for each of the involved constructs. Then, we describe the methodology of our literature review including search, selection, and analysis of relevant publications, before moving on to the presentation of our results. We then discuss our findings and highlight potential limitations of our review. Finally, we close with a concluding statement on our findings and outline avenues for future research.

\section{Theoretical background}

Social media. Social media enables the creation of content, which allows companies to contact social media users and to communicate with them (e.g., [17]). Thus companies can strengthen relations with their customers and build new relations with potential customers (e.g., [61]). In the context of our study, we focus on social media platforms providing companies with the possibility to publish so- called marketergenerated content such as Facebook or Twitter.

Content engagement. In addition to the conceptualization as a psychological state (e.g., [9]), engagement can be a synonym for the interaction with content, which is the consequence of psychological processes (e.g., [16]). Such a conceptualization has, for example, been proposed by van Doorn et al. [56], who identified five essential dimensions of customer engagement behavior, including the valence of the response (i.e., positive or negative feedback) and contextual factors (e.g., where and when the feedback is placed).

Though the psychological processes (i.e., attitudes and behavioral intentions involved in the formation of engagement behaviors) are important to get a full understanding of the causal process behind the content/engagement relationship, we follow the conceptualization by van Doorn et al. [56] and focus on behavioral results (i.e., actual interaction, such as Likes). The main argument for this conceptualization is practical significance as actual behaviors are a more straightforward measurement point for businesses if compared to antecedents of actual behavior. For the purpose of our review, we therefore conceptualize content engagement as the

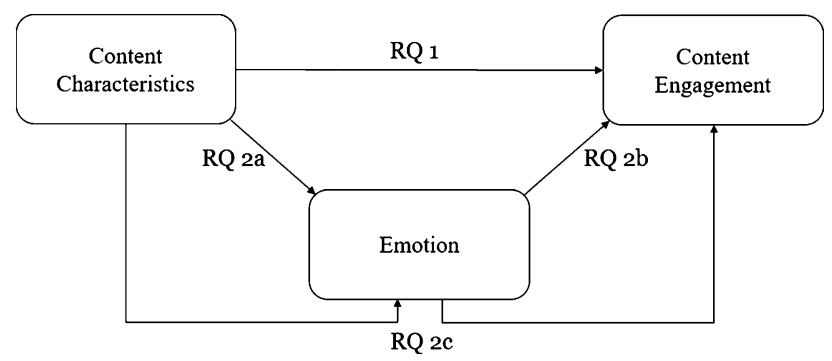

Fig. 1 Research model involving content characteristics, emotions, and content engagement 
measurable results of individual interaction with content on social media channels. Importantly, such a focus does not imply that behavior antecedents such as attitudes, behavioral intentions, or even neurophysiological processes (see, for example, [43]) are generally less important objects of study.

Content characteristics. In a communication process, content usually refers to a message that the sender directs towards potential receivers. A way to further break down the structure of a communication process, which was also used in the context of research on social media before (e.g., [29]), was proposed by Lasswell [31] who stated that the answers to the following questions represent the main characteristics of an act of communication: (1) who, (2) says what, (3) in which channel, (4) to whom, (5) with what effect?

To contextualize our review, we already answered the main part of these questions, as we are interested in corporate communication (1), in the context of social media (3), leading to engagement (5). As we are mostly interested in general effects, we do not narrow down the target group (4) of this communication process and maintain the view that social media communication can be broadly targeted at every individual with Internet access.

When we refer to content characteristics, we are now particularly interested in the remaining element (2) "says what", and extend it by adding "in which context". We are therefore particularly interested in those parts of a social media post that can be manipulated by the sender during its creation. Hence, not only is the actual topic and the way it is presented included here (i.e., the "what" of the content), but we also consider design features such as whether pictures are included or not, or how many lines of text are included. Moreover, we consider the context of the message (e.g., when it was posted or whether a particular position in a stream of messages was chosen for this particular message).

Emotions. It is particularly difficult to define "emotion" as there are other closely related affective concepts, such as preferences or moods [45]. Hence, instead of a unified definition, a model that has garnered much interest, particularly by introducing a classification scheme rather than a general definition of emotions, is the Circumplex Model of Affect proposed by Russell [44]. This model, which has also been applied to the social media context before (e.g., [64]), uses the two dimensions of valence (positive vs. negative) and arousal (arousing vs. relaxing) to classify affective states. The basic premise of this model is that emotions are the outcome of an appraisal process (i.e., a stimulus is processed, which then leads to an observable affective response). In this paper, we focus on the observable part of emotional processing, though we retain the general classification using valence and arousal.

Emotions have already been recognized to have an essential influence on human behaviour in the marketing context. For example, Bagozzi et al. [3] stressed emotions as markers, mediators and moderators of human responses when conducting a literature review. As such, the emotional context of an ad (e.g., happy or sad TV program) effects the evaluation of these ads and their recall [18]. Conducting an experiment using FMRI to capture brain activity and self-reports, Bakalash and Riemer [4] found that the memorability of an ad is positively associated with the elicited emotional arousal. Similarly, Berger and Milkman [7] could confirm that content (online news articles) that evokes high-arousal positive (awe) or negative 
(anger or anxiety) emotions is more viral and engaging (i.e., gets shared more often by email) by following a multimethod approach (i.e., content analysis and experiment).

\section{Methodology of the literature review}

Literature search and selection. We conducted our literature review based on the guidelines by Webster and Watson [60] and vom Brocke et al. [58]. Therefore, we first created a list of keywords based on landmark publications (e.g., [14]) and more recent research (e.g., [15]) (e.g., content engagement, emotional engagement, affective engagement, marketing communication, content emotion, social media, social network, marketing; see online appendix for details). We used keyword combinations to search for relevant publications in a total of 125 peer-reviewed journals and 6 peer-reviewed conference proceedings (see online appendix for details).

Our literature search and selection process consisted of four steps. The first step, using the aforementioned keywords and outlets for an initial search, resulted in a total of 4746 records in journals and conference publications. By evaluating the title and abstract in a first screening phase (done by the first author), we excluded 4611 records, as they were not thematically related to our research questions. In a second step, we removed 85 duplicate records from the remaining 135 publications, which left us with 50 publications for a full-text analysis. The full-text analysis in the third step involved a more detailed look at the relevance of a publication for our research questions (e.g., exclusion of studies as they do not consider the social media context; exclusion of studies as they do not consider our focal constructs; exclusion of studies that do not focus on marketer-generated content-initially done by the first author and discussed with the second and third author). This step resulted in 31 publications being removed. In a fourth step, the remaining 19 publications were used as the basis for a backward and forward search as recommended by Webster and Watson [60], using Google Scholar for the latter part. Through this procedure, we identified an additional 26 publications that are relevant to our research context. Hence, we were left with a sample of $\mathrm{N}=45$ studies for our literature review (see online appendix for details).

Content characteristics. To synthesize previous literature, we used the categories listed below for classification (see online appendix for details). To develop this classification scheme, we used previous research that has already introduced related categories such as topic, length or interactivity (e.g., [47]). It has to be noted that the topic category in particular comprises a broad set of characteristics, which led us to split it up further. The main topic category, includes the type of content such as announcements (e.g., [12]), promotions (e.g., [47]) or messages with emotional (e.g., [35]), functional or informational message appeal (e.g., [53]). The component category includes additional elements that are used to, for example, increase media richness, not necessarily related to a certain topic (e.g., video, picture). In the overview of our classification results (see online appendix for details), for each content characteristic we highlight the publications which included at least one indicator to measure them. 
- (A) Topic: We included indicators for the thematic orientation of a social media post-Example: Posts about specific events or celebrities that appear as testimonials for the company or brand (e.g., $[19,65])$.

- (B) Component: We included indicators of the media richness of a social media post-Example: Other components in addition to text, such as pictures, links, emojis, or hashtags. (e.g., [23, 35]).

- (C) Length: We included indicators of the expansion extensiveness of a social media post-Example: The number of lines of text or its visual height (e.g., $[14,47])$.

- (D) Interactivity: We included indicators of social media posts that actively call for interaction-Example: Requests for customer feedback or raffles with the chance of winning a prize (e.g., [14, 47]).

- (E) Shared or original content: We included indicators for the originality of a social media post-Example: Whether a post was created by the sender or whether it was drawn from a source and reposted (e.g., [32]).

- (F) Timing: We included indicators for the time-related context for a social media post-Example: The time when it was created (e.g., a specific weekday) or the frequency in which new content is added to a social media channel (e.g., [42, 53]).

- (G) Position: We included indicators for the prioritization of a social media post in a communication stream-Example: When a post is "pinned" to the top of a news channel rather than being listed within the typical chronological order of posts (e.g., $[14,47])$.

Emotion. In line with our working definition of emotional responses, we assigned studies to this category if they measured emotional responses on the individual level. This includes psychological metrics (e.g., arousal and valence measured via self-assessment, e.g. [40]), physiological measures (e.g., valence measured via EEG, [30]; see also [39]) or behavioral measures (e.g., facial expression via facial recognition, [33]). It is important to note here, that this excludes analyses of affect, which do not involve individual reactions, such as sentiment analyses of social media posts (e.g., [50]).

Content engagement. In line with our working definition of content engagement, we classified studies which included at least one type of metric of interaction with social media content. This included, for example, liking social media posts (e.g., [51]), sharing a social media post (e.g., [40]), or commenting on a social media post (e.g., [14]). Importantly though, we did not classify studies that indicated engagement intention or comparable constructs that are antecedents of behavior as the focus of our research is on actual behavior (liking, sharing, commenting, etc.). Due to platform specifics (e.g., Twitter), some studies used alternative measures such as favorites (e.g., [41]) or post clicks [16] to capture engagement; we classified these measures as "other" forms of engagement. 


\section{Results}

An initial result of our literature review is the distribution of reviewed studies related to the relationships that we have investigated, which indicates the relative interest in our two research questions and the respective sub-research questions (Fig. 1). Out of our 45 reviewed studies, 39 investigated the content/engagement relationship (RQ 1), with 13 of them including emotion as a content characteristic (e.g., sentiment of a social media post), though not measuring it on the individual level. Six studies focused on the impact of emotional responses in this category and particularly on the effects of content characteristics on emotional responses (RQ 2a). The link between emotional responses and content engagement (RQ 2b) has only been investigated in two studies. Only one study investigated the mediating effect of emotion (RQ 2c). Overall, the studies focused mainly on the social media platform Facebook (31) and considered hardly any other medium (Twitter (7), YouTube (3), Instagram (1), Weibo (1), Taobao (1), Groupon (1)). Four studies mentioned no specific focus at all. Please note that the assignment of studies to research questions and medium is overlapping and not mutually exclusive.

\subsection{Which content characteristics have been shown to be positively related to content engagement? (RQ 1)}

Of the seven categories of content characteristics that we focused on during our literature analysis (A...Topic to G...Position-see online appendix for details), most studies included at least one indicator for the topic of a social media post (i.e., 34 studies). In addition, the use of components, which may enhance the media richness of a social media post was investigated frequently (i.e., 27 studies), all other categories received far less attention.

The topic ( $A-34$ studies) of a social media post has received by far the most attention in our reviewed studies, which can be expected as this category was purposefully defined in a wider fashion, to encompass the large variety of topics that can be dealt with in a social media post (e.g., from general announcements to social media posts with the explicit goal of promoting a company's brand or sales, [46]). The main focus in this category has been on the potential message (i.e., the appeal) that is transported through a social media post. In most cases, studies focused on one of five appeal categories including (i) providing information (e.g., providing information about a company's services and products), (ii) providing entertainment, (iii) transformational messages (e.g., content that addresses the recipient's self-esteem and potential desires), (iv) transactional (e.g., content related to sales, such as discount opportunities), and (v) emotional (e.g., content evoking romantic feelings) (e.g., $[13,27])$.

Some studies in our sample provided evidence for a weak impact of informational appeal (e.g., data about the company, its history, etc.) on content engagement (e.g., [13, 26]). However, most other studies (e.g., [14, 52]) did not find such an effect. Content that was aimed at entertaining its recipients (e.g., funny 
videos) has been shown to positively impact engagement behaviors in most studies (e.g., $[13,26]$ ), but not in all studies that investigated this relationship (e.g., [14]). Transformational content that directly addresses certain needs or desires [27] has been shown to be effective in eliciting engagement (e.g., [11]). Further, Gavilanes et al. [16] and Kim et al. [28] found a positive association between the use of transactional content and content engagement, particularly for announcements of sales or promotions (e.g., discounts, giveaways, etc.). Yet, Schultz [46, 47] found a contradictory effect for transactional content that included references to competitors (e.g., price comparisons with competitors could lead to a negative effect on engagement). Emotional appeal was mainly investigated by using secondary data and then coding it applying a set of discrete emotion categories (e.g., fear, humor, romance, [51, 53]). Some studies found an impact of emotional appeal of textual content (e.g., [50]) or video content [49] on behavioral engagement.

For the use of specific content components ( $B-27$ studies) (e.g., links, text, videos), we found diverging results, regarding the effect of different components and the specific target of this effect (e.g., whether likes or shares were positively affected). Whereas some studies found photo content to receive more engagement (e.g., [28]), others reported the same for video content (e.g., [14]). In addition, similarly to the topic category, we also found diverging results for specific types of engagement behavior (e.g., like or share). For example, Cvijikj and Michahelles [13] found that including videos enhanced like and share behavior, but it did not have any significant effect on the likelihood that users commented on a post. For components more specific to the social media context, such as hashtags and mentions [23], it was found that the use of hashtags can increase engagement slightly [47].

In our sample, one study reported an effect of content length ( $C-10$ studies) on engagement. Wagner et al. [59] found that a complete lack of text or an overabundance of text are less effective in evoking content engagement compared to a moderate amount of text.

For interactivity ( $D-17$ studies), the studies in our sample indicate that including at least one interactive element (e.g., a question or a call for user feedback) can enhance content engagement (e.g., [46]). Yet, the effects of these elements differ strongly. For example, while a contest is more likely to affect "like" behavior, questions are more likely to lead to engagement via comments (e.g., [14, 16]).

Regarding whether social media content was original or shared (E-3 studies), previous research revealed that reposting content that was originally created by users can positively impact content engagement [21], but, in general, original content is more likely to evoke engagement behavior than shared content [27].

For timing ( $F-18$ studies), one study in our sample found a significant effect of weekdays versus weekend on engagement, with content generating more engagement when published on days other than the weekend [13]. Other studies that also included timing as a variable found no significant effects [47].

All of the studies in our sample that investigated the effect of the position $(G-5$ studies) of a social media post within a content stream (e.g., [14, 47], found a small, positive effect on engagement behaviors. 


\subsection{Which effects do emotions have in the context of content characteristics and content engagement? (RQ 2)}

Seven studies investigated the effect of emotions in the content/engagement relationship (RQ 2). According our research model (Fig. 1) we assigned them to (i) the effect of content characteristics on emotional responses (6 studies) (RQ 2a), (ii) the effect of emotional responses on engagement behavior (2 studies) (RQ 2b), and (iii) the relationship among all three construct areas (1 study) (RQ 2c) (see online appendix for details).

Effects of content characteristics on emotional responses (RQ 2a). A first group of studies investigated the effect of video ads on emotional responses. The first study in this group by Teixeira et al. [55] investigated whether emotional responses (i.e., joy and surprise, assessed using facial recognition software) elicited by videos could affect a number of dependent variables (e.g., retention of the video, attention during the video). Yet, they did not explore, which specific content characteristics are involved in the formation of these emotional responses and instead selected videos that they themselves classified as neutral or emotional. Shehu et al. [48] also focused on the emotional response to video content, but they used a self-report scale (ranging from "do not like at all" to "like very much") so participants could evaluate a video when they watched it. Yet, they also did not report on specific content characteristics that could be related to the likability of a video ad.

Lewinski et al. [33] conducted an online experiment to examine the emotional responses to content with a supposedly emotional appeal. The stimulus material consisted of six videos varying in perceived amusement (low, medium or high). Using facial expressions (assessed via Facereader software), they classified the reactions into discrete emotion categories (i.e., happiness, sadness, anger, surprise, fear, and disgust). They only found that facial expressions which were classified as an indicator of happiness (e.g., smiling) could be used to distinguish between amusing and non-amusing videos (in high and medium conditions, not in the low amusement condition).

In addition to the positive emotional response evoked by humor (i.e., highly positive valence), Brown et al. [10] found that the combination with high levels of arousal and potential negative valence, evoked by violence, can have crossover effects. They conducted an online experiment, which involved humorous video ads that varied in their level of violence intensity/severity (e.g., a fictional soft drink commercial with a person being hurt to varying degrees so another person can get to his soft drink). Violence intensity, severity and perceived humor were measured using self-report scales and it was found that perceived humor ameliorates the potential negativity that might be a side-effect of violence depicted in ads.

Aside from video content and emotional appeal, some studies also investigated the emotional response to content with transactional appeal. Yu [64] performed a content analysis to examine the effect of content high in interactivity (e.g., using greetings/wishes from the personified brand, questions aiming to engage) and with a transactional appeal (e.g., product advertising, sales promotion) on engagement. Using a semantic differential scale with six adjective pairs (i.e., happy/unhappy, pleased/annoyed, content/melancholic, hopeful/despairing, satisfied/unsatisfied, 
and relaxed/bored, based on the valence and arousal conceptualization of the affective space by [38]), 13 independent coders classified the emotions evoked by the content. They found that highly interactive content, that is using a personalization of the brand, was perceived as more arousing and received more positive valence ratings than transactional content intent on promoting certain products or sales opportunities.

Transactional appeal should be investigated more thoroughly, as it can have positive effects as well, as shown by Kuan et al. [30]. In their laboratory experiment, they showed product ads consisting of text and pictures to participants, which were accompanied either by additional information or by testimonials (i.e., number of people who bought the product and number of people who liked the product), or by both or by none (control). The emotional effect (i.e., valence) of these variations was measured using the Emotiv EPOC device (an EEG headset with 14 channels). They found that "buy" information alone reduced valence, whereas the addition of "like" information positively affected valence and "like" information alone as an addition to the product ad led to the most positive effect on valence.

Effects of emotional responses on engagement behavior ( $R Q 2 b)$. Only two studies in our sample focused on the relationship between emotional responses and engagement behavior (i.e., [40, 64]). Nelson-Field et al. [40] used a sample of 800 video ads from Facebook (commercial as well as non-commercial), which were coded by 28 raters using a list of 16 discrete emotion categories (i.e., hilarity, amusement, disgust, discomfort, inspiration, calmness, sadness, boredom, astonishment, surprise, shock, irritation, exhilaration, happiness, anger, frustration). These discrete emotions were then recoded into specific arousal and valence ratings. In general, high arousal videos were found to be shared twice as often as low arousal videos and positively valenced/high arousal videos were shared about $30 \%$ more than negatively valenced/high arousal videos. Importantly, they found that valence of a video alone did not lead to any statistically significant effects, with high levels of arousal being a precondition for different sharing behaviors. Yu [64] investigated the effect of different levels of arousal and valence evoked by brand posts on the propensity of an individual to like, share, or comment on a post. While high levels of arousal and or positive valence were positively related to the liking or sharing of a brand post, no such effects were found for the engagement via a comment on the post.

Evidence for the mediating effect of emotional responses $(R Q 2 c)$. Although there has been individual evidence for the relationship between content characteristics and emotional responses (RQ 2a) and emotional responses and engagement behaviors (RQ 2b), we only identified one study in our sample that included indicators for all three areas (i.e., [64]). In this study, Yu evaluated the emotional response based on a content analysis where the emotional perception of content was rated in terms of emotional arousal and valence using two six-item semantic differential scales. They found that social content (e.g., greetings, wishes or questions) is more arousing and positively valenced than promotional content (e.g., advertising, sales promotion or business information). Consequently, users who are more pleased and aroused would rather engage with the content. Yet, in this study a mediating effect was not statistically evaluated, which points to an existing research gap. 


\section{Discussion and research agenda}

Our review of the literature on the relationship between content, emotions and engagement resulted in a number of insights and opportunities for research that we want to point out. For this purpose, we first summarize the main findings for each of our research questions below.

$R Q 1$. Which content characteristics have been shown to be positively related to content engagement? We found that particularly the appeal of a social media post and high media richness (e.g., the inclusion of components such as pictures or videos) can have a positive effect on engagement behaviors. Yet, these results are often not conclusive and highly context dependent (e.g., the content strategy used by the focal company). In addition, we found evidence for mediating effects. For example, content including the chance for some sort of remuneration (e.g., posts that include a raffle) has been shown to have a negative effect on likes but at the same time may have a positive effect on comments [13]. As another example, Luarn et al. [36] found that incentivized content generated more "like" behavior than entertaining content, while entertaining content evoked more sharing behavior than incentivized content.

$R Q 2 a$. Which effects do content characteristics have on emotions? The main findings related to this question result from the analysis of the emotional responses to video content (4 studies). All of them used pre-selected stimulus material in an experimental setting. Yet, several ways to measure emotional response were applied, and it is therefore challenging to compare the results. Only two studies considered further content types and their effect on emotional responses. The contradictory findings of content with transactional appeal could be attributed to the different social media platforms that were used as medium. While Yu [64] analyzed content effects on Facebook, Kuan et al. [30] focused on content from Groupon, a group buying platform, which has a specific focus on transactional content.

$R Q 2 b$. Which effects do emotions have on content engagement? Although engagement was operationalized differently and content types did vary, emotional arousal seems to enhance engagement behavior whereas emotional valence interacts as a facilitator.

$R Q 2 c$. Has there been research into the mediating effect of emotions between content characteristics and content engagement? No, so far this relationship has not been investigated systematically.

These descriptive results of our literature review highlight two main areas for future research: Research Area 1-Investigations to resolve contradictory findings related to the relationship between content characteristics and content engagement and Research Area 2-Further investigations into the effects of emotional responses in the relationship between content characteristics and content engagement.

Research Area 1 (Research Consolidation). Based on the communication process as outlined in the communication model by Lasswell [31], we should look for the reasons for contradictory findings in three main areas: (i) sender, (ii) receiver, or (iii) medium. We want to point out that in each of these areas, researchers should carefully argue for the choices related to their research design and consider its benefits and remedies. 
Sender. With many studies in our review being specific to a certain industry (e.g., $[10,52])$, we argue that a lack in generalizability has led to very limited comparability of studies on social media content engagement. This can hamper further investigations, particularly from a theoretical perspective and we therefore call for more research focusing on the generalizability of results to varying industries.

Receiver. We argue that the analysis of the behavior of an anonymous mass of receivers (as common in content analysis approaches) can lead to dubious insights. As common in the social sciences, control variables that might influence focal constructs have to be specified beforehand, such as demographic (e.g., country of origin, [26]) or psychosocial factors (e.g., attitudes and motivations, [36]) and related data has to be collected. Social media research is not excluded from this requirement and we argue that, even if content analysis is a comfortable way to collect and analyze data whether it can actually form the basis for rigorous research should be reconsidered.

Medium. As social media platforms have different intended purposes (e.g., [25]), the content/engagement relationship will also manifest itself differently within each platform. Several researchers have called for the analysis of data from various platforms in order to get insights on engagement behavior that are more generalizable (e.g., $[26,36])$. We follow this call for generalization and add that if an analysis of several platforms is not possible (e.g., due to resource constraints), those selected should be rigorously argued for and the consequences of this selection should be explained, ideally including research propositions regarding a transfer of the results to another platform.

Research Area 2 (Emotional Effects). Next to highlighting the scarcity of research on the emotional effects of social media content, we want to highlight that insights from marketing and information system research could help us to further substantiate the link between emotional responses and content engagement and could serve as a guide for future research.

In the information systems field, emotions have been prominent for a considerable amount of time. For example, Venkatesh [57] stressed the impact of emotion on technology usage by extending the TAM (technology acceptance model). They conducted three longitudinal field studies and used self-reports to acquire data on categorical emotional response. The results show that perceived enjoyment has an impact on the perceived ease of use and in consequence affects the behavioral intention to use. Similarly, based on a survey to capture categorical emotional response (e.g., excitement, happiness, anger, and anxiety) Beaudry et al. [6] discovered that excitement (i.e., high arousal) has a positive impact on task adaption and IT use.

In addition, previous research on human decision behavior in online auctions could provide us with valuable insights on the potential mediating effect of emotional responses between content and engagement. For example, Adam et al. [1] conducted two experiments and captured arousal via skin conductance and selfreport and valence via heart rate. Following a mediation analysis approach [24] they confirmed a mediating effect of arousal on the relationship between time pressure and bidding behavior as well as the relationship between social competition and bidding behavior. Using such a research process as an example, we argue that a study design framework could be valuable for social media research, 
particularly when it comes to investigating the mediating effects of constructs such as valence and arousal in the relationship between content and engagement.

We summarize our research agenda in Table 1 and, finally, we also want to highlight the limitations of our review, which can be opportunities for future research. First, although we included a large number of publication outlets in our review, we cannot completely eliminate the potential of publication bias. In addition, for the sake of greater clarity in our research goals we mostly used narrow definitions for our focal constructs. We resorted to the definition of engagement as an observable outcome of interaction with content, though there are other conceptualizations of this construct (e.g., [54]). We also resorted to an investigation of emotional responses only although there are other affective constructs (e.g., moods) that could be interesting in the context of social media content and engagement (e.g., [3]). In general, our specific focus on content characteristics that can be manipulated by the sender also leaves further room for extension.

\section{Conclusion}

Based on a review of $\mathrm{N}=45$ studies focusing on the relationship between content characteristics and engagement behaviors in the context of social media, we found initial indications for research investigating the mediating effects of emotional responses. Yet, results are still only partly conclusive and it would thus be particularly worthwhile to use a classification of content, such as the one proposed in this review, to compare social media content regarding its effect on emotional responses and engagement behaviors, which will make future investigations more comparable. In line with Gregor et al. [20], we also call for further investigations in this context, using multi-method designs and a dimensional conceptualization of emotional responses, to create a more elaborate understanding of the relationship we initially depicted in Fig. 1. Furthermore, we recommend future research to use social media communication models as a framework in order to control for confounders and to motivate their research designs.

Table 1 Research agenda

\begin{tabular}{ll}
\hline Research focus & Future research areas \\
\hline $\begin{array}{l}\text { Content characteristics } \\
\text { on content engage- } \\
\text { ment }\end{array}$ & $\begin{array}{l}\text { 1. Effects of content characteristics on engagement in various industry contexts } \\
\text { platforms }\end{array}$ \\
& $\begin{array}{l}\text { 3. Consideration of receiver characteristics as control variables } \\
\text { 4. Development of a communication model for the social media context } \\
\text { Emotional }\end{array}$ \\
$\begin{array}{l}\text { 1. Emotional effects of content characteristics on content engagement } \\
\text { 2. Marketing and information systems research could provide results and } \\
\text { research methods that further elaborate on the effect of emotions }\end{array}$ \\
\hline
\end{tabular}


Acknowledgements Open access funding provided by University of Applied Sciences Upper Austria.

\section{Compliance with ethical standards}

Conflict of interest On behalf of all authors, the corresponding author states that there are no conflicts of interest.

Open Access This article is distributed under the terms of the Creative Commons Attribution 4.0 International License (http://creativecommons.org/licenses/by/4.0/), which permits unrestricted use, distribution, and reproduction in any medium, provided you give appropriate credit to the original author(s) and the source, provide a link to the Creative Commons license, and indicate if changes were made.

\section{References}

1. Adam, M. T. P., Krämer, J., \& Müller, M. B. (2015). Auction fever!: How time pressure and social competition affect bidders' arousal and bids in retail auctions. Journal of Retailing, 91(3), 468-485. https://doi.org/10.1016/j.jretai.2015.01.003.

2. Ashley, C., \& Tuten, T. (2015). Creative strategies in social media marketing: An exploratory study of branded social content and consumer engagement. Psychology and Marketing, 32(1), 15-27.

3. Bagozzi, R. P., Gopinath, M., \& Nyer, P. U. (1999). The role of emotions in marketing. Journal of the Academy of Marketing Science, 27(2), 184-206. https://doi.org/10.1177/0092070399272005.

4. Bakalash, T., \& Riemer, H. (2013). Exploring ad-elicited emotional arousal and memory for the ad using fMRI. Journal of Advertising, 42(4), 275-291. https://doi.org/10.1080/00913367.2013.76806 5 .

5. Baron, R. M., \& Kenny, D. A. (1986). The moderator-mediator variable distinction in social psychological research: Conceptual, strategic, and statistical considerations. Journal of Personality and Social Psychology, 51(6), 1173-1182. https://doi.org/10.1037/0022-3514.51.6.1173.

6. Beaudry, A., \& Pinsonneault, A. (2010). The other side of acceptance: Studying the direct and indirect effects of emotions on information technology use. MIS Quarterly, 34(4), 689-710. https://doi. org/10.2307/25750701.

7. Berger, J., \& Milkman, K. L. (2012). What makes online content viral? Journal of Marketing Research, 49(2), 192-205. https://doi.org/10.1509/jmr.10.0353.

8. Brettel, M., Reich, J.-C., Gavilanes, J. M., \& Flatten, T. C. (2015). What drives advertising success on Facebook? An advertising-effectiveness model: Measuring the effects in sales of "likes" and other social-network stimuli. Journal of Advertising Research, 55(2), 162-175. https://doi. org/10.2501/JAR-55-2-162-175.

9. Brodie, R. J., Hollebeek, L. D., Juric, B., \& Ilic, A. (2011). Customer engagement: Conceptual domain, fundamental propositions, and implications for research. Journal of Service Research, 14(3), 252-271. https://doi.org/10.1177/1094670511411703.

10. Brown, M. R., Bhadury, R. K., \& Pope, N. K. L. (2010). The impact of comedic violence on viral advertising effectiveness. Journal of Advertising, 39(1), 49-66. https://doi.org/10.2753/JOA00 91-3367390104.

11. Coursaris, C., van Osch, W., Balogh McKay, B. A., \& Quilliam, E. T. (2014). Social media marketing: Investigating empirical links between purchase involvement, strategy, content, and media type. In Proceedings of the conference of the american academy of advertising, Atlanta (GA), USA (pp. 131-139).

12. Cvijikj, I. P., \& Michahelles, F. (2011). A case study of the effects of moderator posts within a Facebook brand page. In Proceedings of the 3rd international conference on social informatics (Socinfo), Singapore (pp. 161-170). https://doi.org/10.1007/978-3-642-24704-0_21.

13. Cvijikj, I. P., \& Michahelles, F. (2013). Online engagement factors on Facebook brand pages. Social Network Analysis and Mining, 3(4), 843-861.

14. de Vries, L., Gensler, S., \& Leeflang, P. S. H. (2012). Popularity of brand posts on brand fan pages: An investigation of the effects of social media marketing. Journal of Interactive Marketing, 26(2), 83-91. 
15. Dessart, L. (2017). Social media engagement: A model of antecedents and relational outcomes. Journal of Marketing Management, 54(2), 1-25.

16. Gavilanes, J. M., Flatten, T. C., \& Brettel, M. (2018). Content strategies for digital consumer engagement in social networks: Why advertising is an antecedent of engagement. Journal of Advertising, 47(1), 4-23.

17. Goh, K.-Y., Heng, C.-S., \& Lin, Z. (2013). Social media brand community and consumer behavior: Quantifying the relative impact of user- and marketer-generated content. Information Systems Research, 24(1), 88-107. https://doi.org/10.1287/isre.1120.0469.

18. Goldberg, M. E., \& Gorn, G. J. (1987). Happy and sad TV programs: How they affect reactions to commercials. Journal of Consumer Research, 14(3), 387-403.

19. Gonzalez-Lafaysse, L., \& Lapassouse-Madrid, C. (2016). Facebook and sustainable development: A case study of a French supermarket chain. International Journal of Retail and Distribution Management, 44(5), 560-582. https://doi.org/10.1108/IJRDM-01-2015-0012.

20. Gregor, S., Lin, A. C. H., Gedeon, T., Riaz, A., \& Zhu, D. (2014). Neuroscience and a nomological network for the understanding and assessment of emotions in information systems research. Journal of Management Information Systems, 30(4), 13-48. https://doi.org/10.2753/MIS0742-1222300402.

21. Ha, S., Kankanhalli, A., Kishan, J. S., \& Huang, K.-W. (2016). Does social media marketing really work for online SMEs?: An empirical study. In Proceedings of the 37th international conference on information systems (ICIS), Dublin, Ireland.

22. Hudson, S., Huang, L., Roth, M. S., \& Madden, T. J. (2016). The influence of social media interactions on consumer-brand relationships: A three-country study of brand perceptions and marketing behaviors. International Journal of Research in Marketing, 33(1), 27-41. https://doi.org/10.1016/j. ijresmar.2015.06.004.

23. Hwong, Y.-L., Oliver, C., van Kranendonk, M., Sammut, C., \& Seroussi, Y. (2017). What makes you tick?: The psychology of social media engagement in space science communication. Computers in Human Behavior, 68, 480-492. https://doi.org/10.1016/j.chb.2016.11.068.

24. Imai, K., Keele, L., \& Tingley, D. (2010). A general approach to causal mediation analysis. Psychological Methods, 15(4), 309-334. https://doi.org/10.1037/a0020761.

25. Kaplan, A. M., \& Haenlein, M. (2010). Users of the world, unite! The challenges and opportunities of social media. Business Horizons, 53(1), 59-68.

26. Khan, I., Dongping, H., Wahab, A., \& Lewandowski, D. (2016). Does culture matter in effectiveness of social media marketing strategy? An investigation of brand fan pages. Aslib Journal of Information Management, 68(6), 694-715.

27. Kim, C., \& Yang, S.-U. (2017). Like, comment, and share on Facebook: How each behavior differs from the other. Public Relations Review, 43(2), 441-449. https://doi.org/10.1016/j.pubre v.2017.02.006.

28. Kim, D.-H., Spiller, L., \& Hettche, M. (2015). Analyzing media types and content orientations in Facebook for global brands. Journal of Research in Interactive Marketing, 9(1), 4-30.

29. Knoll, J. (2016). Advertising in social media: A review of empirical evidence. International Journal of Advertising, 35(2), 266-300. https://doi.org/10.1080/02650487.2015.1021898.

30. Kuan, K. K. Y., Zhong, Y., \& Chau, P. Y. K. (2014). Informational and normative social influence in group-buying: Evidence from self-reported and EEG data. Journal of Management Information Systems, 30(4), 151-178. https://doi.org/10.2753/MIS0742-1222300406.

31. Lasswell, H. D. (1948). The structure and function of communication in society. The Communication of Ideas, 37, 215-228.

32. Lee, J., \& Hong, I. B. (2016). Predicting positive user responses to social media advertising: The roles of emotional appeal, informativeness, and creativity. International Journal of Information Management, 36(3), 360-373. https://doi.org/10.1016/j.ijinfomgt.2016.01.001.

33. Lewinski, P., Fransen, M. L., \& Tan, E. S. H. (2014). Predicting advertising effectiveness by facial expressions in response to amusing persuasive stimuli. Journal of Neuroscience, Psychology, and Economics, 7(1), 1-14. https://doi.org/10.1037/npe0000012.

34. Lin, R., \& Utz, S. (2015). The emotional responses of browsing Facebook: Happiness, envy, and the role of tie strength. Computers in Human Behavior, 52, 29-38. https://doi.org/10.1016/j. chb.2015.04.064.

35. Liu, J., Li, C., Ji, Y. G., North, M., \& Yang, F. (2017). Like it or not: The fortune 500's Facebook strategies to generate users' electronic word-of-mouth. Computers in Human Behavior, 73, 605613. https://doi.org/10.1016/j.chb.2017.03.068. 
36. Luarn, P., Lin, Y.-F., \& Chiu, Y.-P. (2015). Influence of Facebook brand-page posts on online engagement. Online Information Review, 39(4), 505-519.

37. Mauri, M., Cipresso, P., Balgera, A., Villamira, M., \& Riva, G. (2011). Why is Facebook so successful? Psychophysiological measures describe a core flow state while using Facebook. Cyberpsychology, Behavior and Social Networking, 14(12), 723-731. https://doi.org/10.1089/cyber.2010.0377.

38. Mehrabian, A., \& Russell, J. A. (1974). An approach to environmental psychology. Cambridge: The MIT Press.

39. Müller-Putz, G. R., Riedl, R., \& Wriessnegger, S. C. (2015). Electroencephalography (EEG) as a research tool in the information systems discipline: Foundations, measurement, and applications. Communications of the Association for Information Systems, 37, 911-948.

40. Nelson-Field, K., Riebe, E., \& Newstead, K. (2013). The emotions that drive viral video. Australasian Marketing Journal, 21(4), 205-211. https://doi.org/10.1016/j.ausmj.2013.07.003.

41. Parganas, P., Anagnostopoulos, C., \& Chadwick, S. (2015). 'You'll never tweet alone': Managing sports brands through social media. Journal of Brand Management, 22(7), 551-568.

42. Pinto, M. B., \& Yagnik, A. (2016). Fit for life: A content analysis of fitness tracker brands use of Facebook in social media marketing. Journal of Brand Management. https://doi.org/10.1057/ s41262-016-0014-4.

43. Riedl, R., \& Léger, P.-M. (2016). Fundamentals of neuroIS: Information systems and the brain (Studies in neuroscience, psychology and behavioral economics). Berlin: Springer.

44. Russell, J. A. (1980). A circumplex model of affect. Journal of Personality and Social Psychology, 39(6), 1161-1178. https://doi.org/10.1037/h0077714.

45. Scherer, K. R. (2005). What are emotions? And how can they be measured? Social Science Information, 44(4), 695-729. https://doi.org/10.1177/0539018405058216.

46. Schultz, C. D. (2016). Driving likes, comments, and shares on social networking sites. In Proceedings of the 18th annual international conference on electronic commerce (ICEC), Suwon, Republic of Korea. ACM Press.

47. Schultz, C. D. (2017). Proposing to your fans: Which brand post characteristics drive consumer engagement activities on social media brand pages? Electronic Commerce Research and Applications, 26, 23-34.

48. Shehu, E., Bijmolt, T. H. A., \& Clement, M. (2016). Effects of likeability dynamics on consumers' intention to share online video advertisements. Journal of Interactive Marketing, 35, 27-43.

49. Southgate, D., Westoby, N., \& Page, G. (2010). Creative determinants of viral video viewing. International Journal of Advertising, 29(3), 349-368. https://doi.org/10.2501/S02650487102012 21.

50. Stieglitz, S., \& Dang-Xuan, L. (2013). Emotions and information diffusion in social media-sentiment of microblogs and sharing behavior. Journal of Management Information Systems, 29(4), 217-248. https://doi.org/10.2753/MIS0742-1222290408.

51. Swani, K., Milne, G., \& Brown, B. P. (2013). Spreading the word through likes on Facebook: Evaluating the message strategy effectiveness of fortune 500 companies. Journal of Research in Interactive Marketing, 7(4), 269-294.

52. Swani, K., \& Milne, G. R. (2017). Evaluating Facebook brand content popularity for service versus goods offerings. Journal of Business Research, 79, 123-133.

53. Swani, K., Milne, G. R., Brown, B. P., Assaf, A. G., \& Donthu, N. (2017). What messages to post? Evaluating the popularity of social media communications in business versus consumer markets. Industrial Marketing Management, 62, 77-87. https://doi.org/10.1016/j.indma rman.2016.07.006.

54. Syrdal, H. A., \& Briggs, E. (2018). Engagement with social media content: A qualitative exploration. The Journal of Marketing Theory and Practice, 26(1-2), 4-22.

55. Teixeira, T., Wedel, M., \& Pieters, R. (2012). Emotion-induced engagement in internet video advertisements. Journal of Marketing Research, 49(2), 144-159. https://doi.org/10.1509/jmr.10.0207.

56. van Doorn, J., Lemon, K. N., Mittal, V., Nass, S., Pick, D., Pirner, P., et al. (2010). Customer engagement behavior: Theoretical foundations and research directions. Journal of Service Research, 13(3), 253-266. https://doi.org/10.1177/1094670510375599.

57. Venkatesh, V. (2000). Determinants of perceived ease of use: Integrating control, intrinsic motivation, and emotion into the technology acceptance model. Information Systems Research, 11(4), 342-365. https://doi.org/10.1287/isre.11.4.342.11872.

58. vom Brocke, J., Simons, A., Niehaves, B., Riemer, K., Plattfaut, R., Cleven, A., et al. (2009). Reconstructing the giant: On the importance of rigour in documenting the literature search process. In AIS 
(Ed.), Proceedings of the 17th European conference on information systems (ECIS 2009), Verona, Italy (Vol. 9, pp. 2206-2217).

59. Wagner, T. F., Baccarella, C., \& Voigt, K.-I. (2016). Antecedents of brand post popularity in Facebook: The influence of images, videos, and text. In Proceedings of the 15th international marketing trends conference (IMTC), Venice, Italy.

60. Webster, J., \& Watson, R. T. (2002). Analyzing the past to prepare for the future: Writing a literature review. MIS Quarterly, 26(2), xiii-xxiii.

61. Weiger, W. H., Wetzel, H. A., \& Hammerschmidt, M. (2017). Leveraging marketer-generated appeals in online brand communities. Journal of Service Management, 28(1), 133-156. https://doi. org/10.1108/JOSM-11-2015-0378.

62. Woodworth, R. S. (1958). Dynamics of behavior. Oxford: Holt.

63. Young, J. (2015). Content marketing will be a $\$ 300$ billion industry by 2019. Marketing Magazine.

64. Yu, J. (2014). We look for social, not promotion: Brand post strategy, consumer emotions, and engagement-A case study of the Facebook brand pages. GSTF Journal on Media \& Communications, 1(2), 32-41. https://doi.org/10.5176/2335-6618_1.2.17.

65. Zhang, Y., Moe, W. W., \& Schweidel, D. A. (2016). Modeling the role of message content and influencers in social media rebroadcasting. International Journal of Research in Marketing, 34(1), 100-119. https://doi.org/10.1016/j.ijresmar.2016.07.003. 